POS PROCEEDINGS

\title{
AZURE, An R-Matrix Computer Code
}

\author{
Richard deBoer and Richard Azuma* \\ University of Notre Dame \\ E-mail: Richard.J.deBoer.12@nd.edu, r.azuma@sympatico.ca
}

C.R. Brune

Ohio University

\section{H. Costantini}

Istituto Nazionale di Fisica Nucleare

\section{J. Görres, P.J. LeBlanc, E. Uberseder, and M. Wiescher}

University of Notre Dame

\section{E.C. Simpson}

University of Surrey

\section{Ugalde}

Triangle University Nuclear Laboratory

\begin{abstract}
The R-Matrix technique [1] has proved to be a powerful tool for the analysis of the nuclear data for the purpose of extracting level information and extrapolation of the astrophysical S-Factor to Gamow energies. With the motivation of creating a comprehensive R-Matrix analysis tool, AZURE [2] was developed. The code distinguishes itself through its multichannel, multilevel, external capture, parameter transformation, and user friendly capabilities.

Transformations developed by Barker [3] and Brune [4] are used to convert from R-Matrix parameters to experimental observables and vice versa. External capture is included because of its importance in low energy radiative decay analysis, and has been implemented using methods derived from Barker and Kajino [5] and Angulo and Descouvemont [6]. In the interest of ease of use, a graphical setup application or Setup Utility has been created and an extensive user manual is planned for release concurrent with publication.
\end{abstract}

10th Symposium on Nuclei in the Cosmos

July 27 - August 12008

Mackinac Island, Michigan, USA

\footnotetext{
* Speaker.
} 


\section{Introduction}

Although R-matrix computer codes have been widely used in nuclear astrophysics, and many such codes are available, we have endeavored to write a general multi-channel, multi-level R-matrix code that is both versatile in its application as well as accessible to a wide range users who are not experts in R-matrix theory.

AZURE is an R-matrix code written in FORTRAN [2]. Its purpose is the calculation of differential and total cross sections of interest in nuclear astrophysics. The R-matrix sections of the code follow the formulation of A.M.Lane and R.G.Thomas [1] ( herein after referred to as LT). The notation of LT is followed whenever possible. The code for the R-matrix cross section calculations is embedded in a MINUIT (CERN) [7] least squares batch mode environment so that the theoretical calculations can be fitted to the experimental data. The Linear Algebra PACKage (LAPACK) [8] is also utilized for matrix inversion in the transformation codes.

\section{R-matrix Concepts}

In using R-matrix theory there are two sets of states to be considered. The 'physical' states are the compound resonance states responsible for the resonances, with parameters $\mathrm{E}_{r}$ and $\Gamma$. However these are neither the states nor the parameters which are used in the R-matrix calculations. The 'Rmatrix' states actually used in the calculations are an infinite set of internal states (LT III), whose parameters are the pole energies $\mathrm{E}_{0}$ and the reduced width amplitudes $\gamma$. In the spirit of LT a correspondence between the two sets is made by associating the lowest energy observed physical states with the corresponding lowest energy R-matrix states, and representing all higher-lying levels with a single back ground state (or pole). The R-matrix energies and reduced width amplitudes are determined in the fit of the theory to the data. The inclusion of the BG state often has a profound effect on the yield curve due to the interferences involved.

As noted, all fits are performed with the internal R-matrix parameters. The corresponding physical parameters appear only after the R-matrix calculations are completed in the output part of the code, where they are calculated from the R-matrix values, using the transformation theory of F.C.Barker [3]. The user may also choose to give parameters corresponding to 'physical' states as the input to AZURE. These will be transformed into R-matrix parameters as input to the R-Matrix calculations. This transformation is based on the paper C.R.Brune [4].

\section{Reactions and Channels}

An R-matrix calculation involves the specification of the entrance channel particle pair, the properties of the compound resonance states, and the enumeration of the exit channel particle pairs for all the open decay reactions allowed by the physics (or chosen by the user).

In the notation LT, the entrance channel is identified by the set of quantum numbers $(\alpha s \ell)$ with corresponding indices $\left(\alpha^{\prime} s^{\prime} \ell^{\prime}\right)$ for the exit particle pairs. Here, $\alpha$ and $\alpha^{\prime}$ identify the particle pairs, while $s\left(s^{\prime}\right)$ and $\ell\left(\ell^{\prime}\right)$ are the channel spins and relative orbital angular momenta respectively. Only one entrance channel particle pair may be defined, whereas as many decay channels as is consistent with the physics (or the choice of the user) may be specified. The angular momentum channels are 
defined by the allowed combinations of the channel spins $s\left(s^{\prime}\right)$ with the relative orbital angular momenta $\ell\left(\ell^{\prime}\right)$ and $\mathbf{J}^{\pi}$ of the compound resonance state. After input of the nuclear information (see below), these channel calculations are performed automatically by AZURE and all channels will be included in a simultaneous calculation, with all physically allowed interferences included.

The External Capture (EC) process is often a significant contribution to the radiative capture cross section at low energies. Therefore EC has been included in AZURE using the techniques of F.C. Barker and T. Kajino [5] and C. Angulo and P. Descouvemont [6]. The strength of the EC can be expressed by AZURE as either a dimensionless reduced width amplitude or an Asymptotic Normalization Coefficient ANC.

\section{Physics Input}

The main input for AZURE is experimental cross section data and nuclear structure information. Cross section data can take the form of differential or angle integrated and can be a function of energy, angle or both. For yield data there are several options for beam convolution and target integration. A Master Index file system has been developed that allows for easy data segmentation and manipulation.

The cross section, nuclear structure and configuration options are entered into several configuration files that are read by AZURE. This initial data entry can be tedious and time consuming so a graphical setup application or Setup Utility was created. The Setup Utility acts as an interpreter between the user and AZURE offering a visual interface for the user and producing data files in the precise format required by FOR-

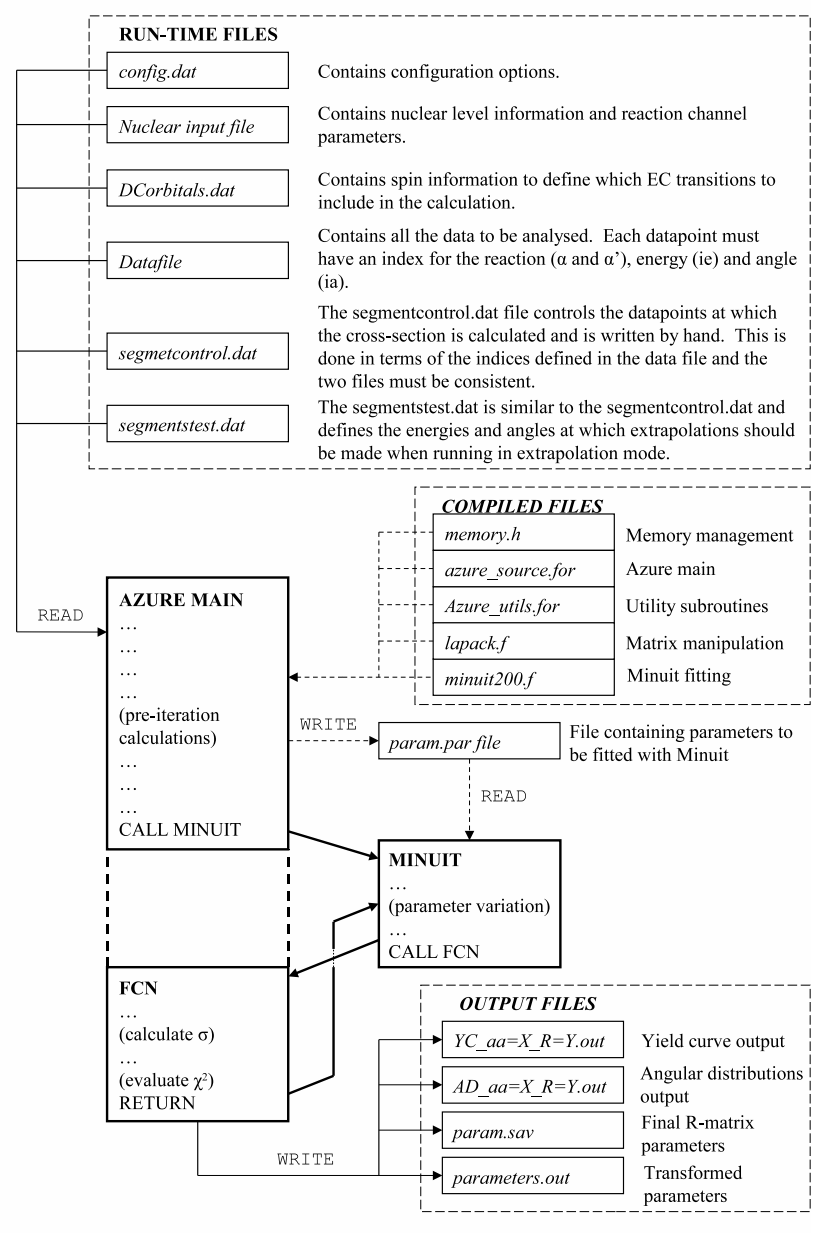

Figure 1: AZURE Code Structure TRAN. The Setup Utility has been found to greatly expedite data entry and option control throughout the use of AZURE. 


\section{Code Structure}

The core of AZURE consists of three main subroutines: AZURE Main, FCN, and MINUIT as shown in Figure 1. This code structure was developed for both utility and efficiency. AZURE Main first calculates all quantities that are independent of the fit parameters $\mathrm{E}_{0}$ and $\gamma$. AZURE Main then passes MINUIT the initial values of the fit parameters. MINUIT and FCN act in concert for the actual fitting and minimization procedure. MINUIT varies the fit parameters and hands them to FCN. FCN calculates the cross section and $\chi^{2}$ which is used as the control parameter to be minimized. $\chi^{2}$ is then passed back to MINUIT and the process repeats until a minimum in $\chi^{2}$ is reached.

To improve efficiency all matrix elements are grouped into the $\left(\alpha s, \alpha^{\prime} s^{\prime}\right)$ indexing scheme of LT for the cross section calculation in FCN. This avoids redundant nested loops over unnecessary quantum numbers which can be very computationly costly since FCN may be called thousands of times during a minimization. This indexing structure is known as reaction pathways since it effectively groups the matrix elements into entrance and exit channel particle pairs.

\section{Examples}

The following fit examples highlight the capabilities of AZURE. When multiple fits are shown for a given example, all fits where done simultaneously.

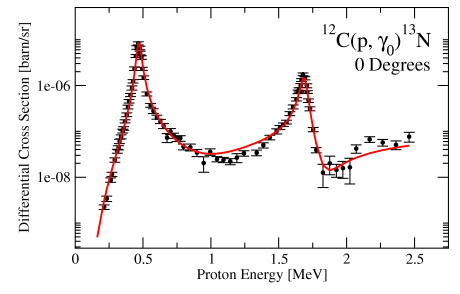

(a) ${ }^{12} \mathrm{C}(\mathrm{p}, \gamma) 0^{\circ}$

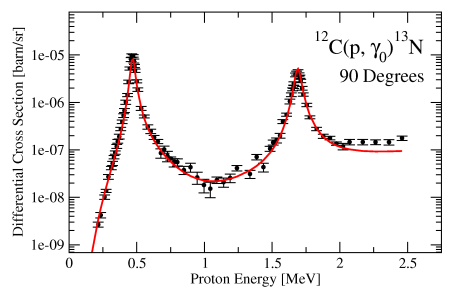

(b) ${ }^{12} \mathrm{C}(\mathrm{p}, \gamma) 90^{\circ}$

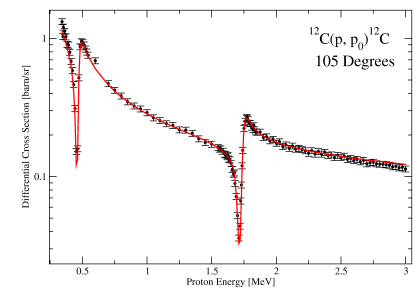

(c) ${ }^{12} \mathrm{C}(\mathrm{p}, \mathrm{p})$

Figure 2: The classic ${ }^{12} \mathrm{C}(\mathrm{p}, \gamma)$ R-matrix analysis of Rolfs and Azuma [9] is redone with the addition of scattering data from Ref. [10].

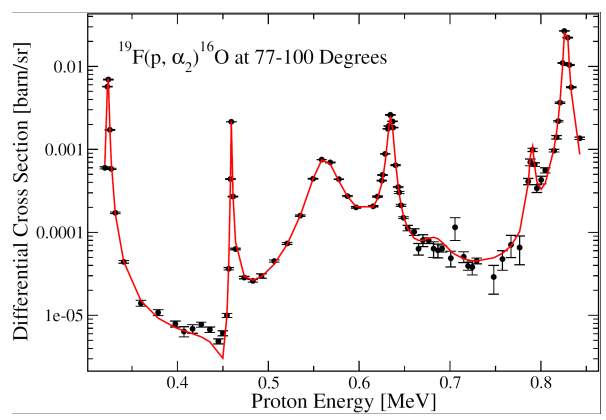

Figure 3: ${ }^{19} \mathrm{~F}\left(\mathrm{p}, \alpha_{2}\right)$ differential cross section data from Ref. [13]. This fit displays the multilevel capability of AZURE with its ability to reproduce complex interference effects. The Master Index file system allows for the direct analysis of this data which is incremented in angle as well as energy. 


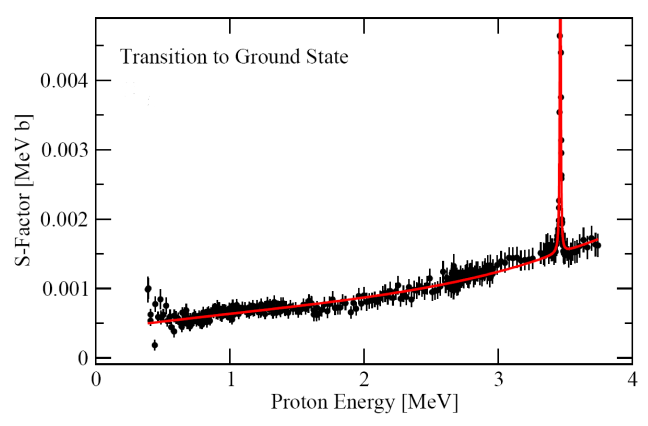

(a) ${ }^{16} \mathrm{O}\left(\mathrm{p}, \gamma_{0}\right)$

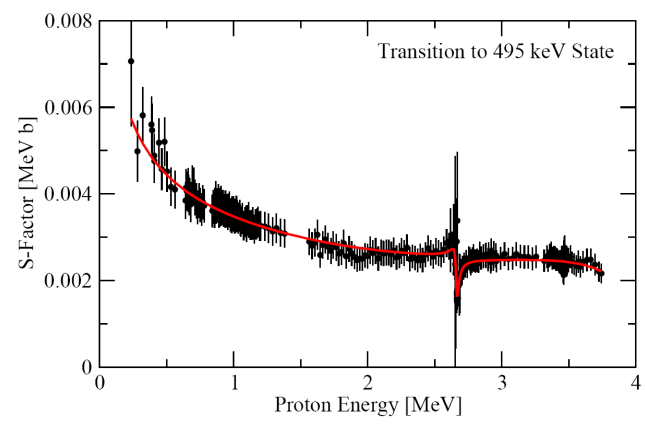

(b) ${ }^{16} \mathrm{O}\left(\mathrm{p}, \gamma_{1}\right)$

Figure 4: Fits to the ${ }^{16} \mathrm{O}(\mathrm{p}, \gamma)$ Direct Capture data of Ref. [11] correct by Ref. [12]. The cross section is dominated by EC.

\section{References}

[1] A.M. Lane and R.G. Thomas, Reviews of Modern Physics 302 (1958)

[2] R.E. Azuma et al., In preparation

[3] F.C.Barker, Aust. J. Phys. 25, 341 (1972)

[4] C.R. Brune, Phys. Rev. C 66044611 (2002)

[5] F.C. Barker and T. Kajino, Aust. J. Phys. 44 369-96 (1991)

[6] C. Angulo and P. Descouvemont, Nuclear Physics A 690 755-768 (2001)

[7] F. James, Minuit: Function Minimization and Error Analysis Reference Manual, Version 94.1,CERN Geneva, Switzerland

[8] E. Anderson et al., LAPACK User's Manual, Version 3.2 (1999)

[9] C. Rolfs and R.E. Azuma, Nuclear Physics A 227 291-308 (1974)

[10] S. Mazzoni et al., Nucl. Instr. Meth. B 136-138 86 (1998)

[11] R. Morlock et al., Phys. Rev. Lett. 79 3837-3840 (1997)

[12] C. Iliadis et al., Phys. Rev. C 77045802 (2008)

[13] R. Ott, Ph.D. thesis, Universität Stuttgart, (1997) 